TRANSACTIONS OF THE

AMERICAN MATHEMATICAL SOCIETY

Volume 353, Number 11, Pages 4447-4460

S 0002-9947(01)02766-0

Article electronically published on May 22, 2001

\title{
SPHERICAL CLASSES AND THE LAMBDA ALGEBRA
}

\author{
NGUYẼ̃N H. V. HUNG
}

Abstract. Let $\Gamma^{\wedge}=\bigoplus_{k} \Gamma_{k}^{\wedge}$ be Singer's invariant-theoretic model of the dual of the lambda algebra with $H_{k}\left(\Gamma^{\wedge}\right) \cong \operatorname{Tor}_{k}^{\mathcal{A}}\left(\mathbb{F}_{2}, \mathbb{F}_{2}\right)$, where $\mathcal{A}$ denotes the mod 2 Steenrod algebra. We prove that the inclusion of the Dickson algebra, $D_{k}$, into $\Gamma_{k}^{\wedge}$ is a chain-level representation of the Lannes-Zarati dual homomorphism

$$
\varphi_{k}^{*}: \mathbb{F}_{2} \underset{\mathcal{A}}{\otimes} D_{k} \rightarrow \operatorname{Tor}_{k}^{\mathcal{A}}\left(\mathbb{F}_{2}, \mathbb{F}_{2}\right) \cong H_{k}\left(\Gamma^{\wedge}\right) .
$$

The Lannes-Zarati homomorphisms themselves, $\varphi_{k}$, correspond to an associated graded of the Hurewicz map

$$
H: \pi_{*}^{s}\left(S^{0}\right) \cong \pi_{*}\left(Q_{0} S^{0}\right) \rightarrow H_{*}\left(Q_{0} S^{0}\right) .
$$

Based on this result, we discuss some algebraic versions of the classical conjecture on spherical classes, which states that Only Hopf invariant one and Kervaire invariant one classes are detected by the Hurewicz homomorphism. One of these algebraic conjectures predicts that every Dickson element, i.e. element in $D_{k}$, of positive degree represents the homology class 0 in $\operatorname{Tor}_{k}^{\mathcal{A}}\left(\mathbb{F}_{2}, \mathbb{F}_{2}\right)$ for $k>2$.

We also show that $\varphi_{k}^{*}$ factors through $\mathbb{F}_{2} \underset{\mathcal{A}}{\otimes} \operatorname{Ker} \partial_{k}$, where $\partial_{k}: \Gamma_{k}^{\wedge} \rightarrow$ $\Gamma_{k-1}^{\wedge}$ denotes the differential of $\Gamma^{\wedge}$. Therefore, the problem of determining $\mathbb{F}_{2} \otimes{ }_{\mathcal{A}}$ Ker $\partial_{k}$ should be of interest.

\section{Introduction AND STATEMENT OF RESULTS}

Let $Q_{0} S^{0}$ be the basepoint component of $Q S^{0}=\lim _{n} \Omega^{n} S^{n}$. It is a classical unsolved problem to compute the image of the Hurewicz homomorphism

$$
H: \pi_{*}^{s}\left(S^{0}\right) \cong \pi_{*}\left(Q_{0} S^{0}\right) \rightarrow H_{*}\left(Q_{0} S^{0}\right) .
$$

Here and throughout the paper, homology and cohomology are taken with coefficients in $\mathbb{F}_{2}$, the field of two elements. The long-standing conjecture on spherical classes reads as follows.

Conjecture 1.1. The Hopf invariant one and the Kervaire invariant one classes are the only elements in $H_{*}\left(Q_{0} S^{0}\right)$ detected by the Hurewicz homomorphism. (See Curtis [5], Snaith and Tornehave [22] and Wellington [23] for a discussion.)

An algebraic version of this problem goes as follows. Let $P_{k}=\mathbb{F}_{2}\left[x_{1}, \ldots, x_{k}\right]$ be the polynomial algebra on $k$ generators $x_{1}, \ldots, x_{k}$, each of degree 1 . Let the

Received by the editors February 4, 1999 and, in revised form, November 4, 1999.

2000 Mathematics Subject Classification. Primary 55P47, 55Q45, 55S10, 55T15.

Key words and phrases. Spherical classes, loop spaces, Adams spectral sequences, Steenrod algebra, lambda algebra, invariant theory, Dickson algebra.

The research was supported in part by the National Research Project, No. 1.4.2. 
general linear group $G L_{k}=G L\left(k, \mathbb{F}_{2}\right)$ and the mod 2 Steenrod algebra $\mathcal{A}$ both act on $P_{k}$ in the usual way. The Dickson algebra of $k$ variables, $D_{k}$, is the algebra of invariants

$$
D_{k}:=\mathbb{F}_{2}\left[x_{1}, \ldots, x_{k}\right]^{G L_{k}} .
$$

As the action of $\mathcal{A}$ and that of $G L_{k}$ on $P_{k}$ commute with each other, $D_{k}$ is an algebra over $\mathcal{A}$. In [14, Lannes and Zarati construct homomorphisms

$$
\varphi_{k}: \operatorname{Ext}_{\mathcal{A}}^{k, k+i}\left(\mathbb{F}_{2}, \mathbb{F}_{2}\right) \rightarrow\left(\mathbb{F}_{2} \otimes D_{\mathcal{A}}\right)_{i}^{*}
$$

which correspond to an associated graded of the Hurewicz map. The proof of this assertion is unpublished, but it is sketched by Lannes 12 and by Goerss [7. The Hopf invariant one and the Kervaire invariant one classes are respectively represented by certain permanent cycles in $\operatorname{Ext}_{\mathcal{A}}^{1, *}\left(\mathbb{F}_{2}, \mathbb{F}_{2}\right)$ and $\operatorname{Ext}_{\mathcal{A}}^{2, *}\left(\mathbb{F}_{2}, \mathbb{F}_{2}\right)$, on which $\varphi_{1}$ and $\varphi_{2}$ are non-zero (see Adams [1], Browder [4], Lannes-Zarati [14]). Therefore, we are led to the following conjecture.

Conjecture 1.2. $\varphi_{k}=0$ in any positive stem $i$ for $k>2$.

The present paper follows a series of our works $([8$, [10], [11]) on this conjecture. To state our main result, we need to summarize Singer's invariant-theoretic description of the lambda algebra [20]. According to Dickson [6], one has

$$
D_{k} \cong \mathbb{F}_{2}\left[Q_{k, k-1}, \ldots, Q_{k, 0}\right],
$$

where $Q_{k, i}$ denotes the Dickson invariant of degree $2^{k}-2^{i}$. Singer sets $\Gamma_{k}=$ $D_{k}\left[Q_{k, 0}^{-1}\right]$, the localization of $D_{k}$ given by inverting $Q_{k, 0}$, and defines $\Gamma_{k}^{\wedge}$ to be a certain "not too large" submodule of $\Gamma_{k}$. He also equips $\Gamma^{\wedge}=\bigoplus_{k} \Gamma_{k}^{\wedge}$ with a differential $\partial: \Gamma_{k}^{\wedge} \rightarrow \Gamma_{k-1}^{\wedge}$ and a coproduct. Then, he shows that the differential coalgebra $\Gamma^{\wedge}$ is dual to the lambda algebra of the six authors of [3]. Thus, $H_{k}\left(\Gamma^{\wedge}\right) \cong$ $\operatorname{Tor}_{k}^{\mathcal{A}}\left(\mathbb{F}_{2}, \mathbb{F}_{2}\right)$. (Originally, Singer uses the notation $\Gamma_{k}^{+}$to denote $\Gamma_{k}^{\wedge}$. However, by $D_{k}^{+}, \mathcal{A}^{+}$we always mean the submodules of $D_{k}$ and $\mathcal{A}$ respectively consisting of all elements of positive degrees, so Singer's notation $\Gamma_{k}^{+}$would cause confusion in this paper. Therefore, we prefer the notation $\Gamma_{k}^{\wedge}$.)

The main result of this paper is the following theorem, which has been conjectured in our paper [10, Conjecture 5.3].

Theorem 3.9. The inclusion $D_{k} \subset \Gamma_{k}^{\wedge}$ is a chain-level representation of the Lannes-Zarati dual homomorphism

$$
\left.\varphi_{k}^{*}: \underset{\mathcal{A}}{\left(\mathbb{F}_{2}\right.} \underset{D_{k}}{\otimes}\right)_{i} \rightarrow \operatorname{Tor}_{k, k+i}^{\mathcal{A}}\left(\mathbb{F}_{2}, \mathbb{F}_{2}\right) .
$$

An immediate consequence of this theorem is the equivalence between Conjecture 1.2 and the following one.

Conjecture 1.3. If $q \in D_{k}^{+}$, then $[q]=0$ in $\operatorname{Tor}_{k}^{\mathcal{A}}\left(\mathbb{F}_{2}, \mathbb{F}_{2}\right)$ for $k>2$.

This has been established for $k=3$ in [10, Theorem 4.8], while Conjecture [1.2 has been proved for $k=3$ in [8, Corollary 3.5] .

From the view point of this conjecture, it seems to us that Singer's model of the dual of the lambda algebra, $\Gamma^{\wedge}$, is somehow more natural than the lambda algebra itself.

The canonical $\mathcal{A}$-action on $D_{k}$ is extended to an $\mathcal{A}$-action on $\Gamma_{k}^{\wedge}$. This action commutes with $\partial_{k}$ (see [20]), so it determines an $\mathcal{A}$-action on $\operatorname{Ker} \partial_{k}$, the submodule of all cycles in $\Gamma_{k}^{\wedge}$. We also prove 
Proposition 4.1. $\varphi_{k}^{*}$ factors through $\mathbb{F}_{2} \otimes K e r \partial_{k}$ as shown in the commutative diagram

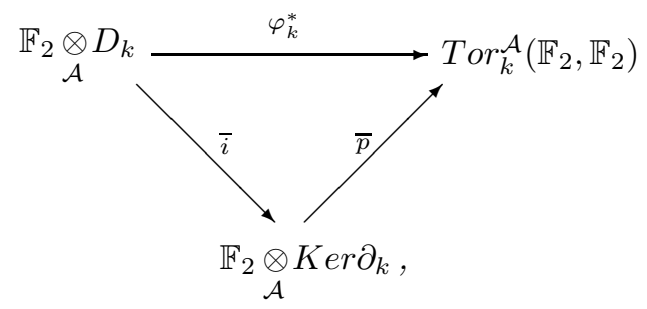

where $\bar{i}$ is induced by the inclusion $D_{k} \subset K e r \partial_{k}$, and $\bar{p}$ is an epimorphism induced by the canonical projection $p: \operatorname{Ker}_{k} \rightarrow H_{k}\left(\Gamma^{\wedge}\right) \cong \operatorname{Tor}_{k}^{\mathcal{A}}\left(\mathbb{F}_{2}, \mathbb{F}_{2}\right)$.

From this result, the problem of determining $\mathbb{F}_{2} \otimes K e r \partial_{k}$ would be of interest.

The paper is divided into 4 sections.

$\mathcal{A}$

In Section 2 we recollect some materials on invariant theory, particularly on Singer's invariant-theoretic description of the lambda algebra and the LannesZarati homomorphism. Section 3 is devoted to prove Theorem 3.9. Finally, Section 4 is a discussion on factoring $\varphi_{k}^{*}$.

The main results of this paper were announced in [9].

The author would like to thank Haynes Miller for introducing him to Stewart Priddy's work [18] on exploiting an explicit homotopy equivalence between the bar resolution of $\mathbb{F}_{2}$ over $\mathcal{A}$ and the dual of the lambda algebra. He also thanks the referee for helpful suggestions, which led to improving the exposition of the paper.

\section{RECOLLECTIONS ON MODULAR INVARIANT THEORY}

We start this section by sketching briefly Singer's invariant-theoretic description of the lambda algebra.

Let $T_{k}$ be the Sylow 2-subgroup of $G L_{k}$ consisting of all upper triangular $k \times k$ matrices with 1 on the main diagonal. The $T_{k}$-invariant ring, $M_{k}=P_{k}^{T_{k}}$, is called the Mùi algebra. In [17, Mùi shows that

$$
P_{k}^{T_{k}}=\mathbb{F}_{2}\left[V_{1}, \ldots, V_{k}\right]
$$

where

$$
V_{i}=\prod_{\lambda_{j} \in \mathbb{F}_{2}}\left(\lambda_{1} x_{1}+\cdots+\lambda_{i-1} x_{i-1}+x_{i}\right)
$$

Then, the Dickson invariant $Q_{k, i}$ can inductively be defined by

$$
Q_{k, i}=Q_{k-1, i-1}^{2}+V_{k} \cdot Q_{k-1, i}
$$

where, by convention, $Q_{k, k}=1$ and $Q_{k, i}=0$ for $i<0$.

Let $S(k) \subset P_{k}$ be the multiplicative subset generated by all the non-zero linear forms in $P_{k}$. Let $\Phi_{k}$ be the localization, $\Phi_{k}=\left(P_{k}\right)_{S(k)}$. Using the results of Dickson [6] and Mùi [17], Singer notes in [20] that

$$
\begin{gathered}
\Delta_{k}:=\left(\Phi_{k}\right)^{T_{k}}=\mathbb{F}_{2}\left[V_{1}^{ \pm 1}, \ldots, V_{k}^{ \pm 1}\right], \\
\Gamma_{k}:=\left(\Phi_{k}\right)^{G L_{k}}=\mathbb{F}_{2}\left[Q_{k, k-1}, \ldots, Q_{k, 1}, Q_{k, 0}^{ \pm 1}\right] .
\end{gathered}
$$

Further, he sets

$$
v_{1}=V_{1}, \quad v_{k}=V_{k} / V_{1} \cdots V_{k-1} \quad(k \geq 2),
$$


so that

Then, he obtains

$$
V_{k}=v_{1}^{2^{k-2}} v_{2}^{2^{k-3}} \cdots v_{k-1} v_{k} \quad(k \geq 2)
$$

with $\operatorname{deg} v_{i}=1$ for every $i$.

$$
\Delta_{k}=\mathbb{F}_{2}\left[v_{1}^{ \pm 1}, \ldots, v_{k}^{ \pm 1}\right]
$$

Singer defines $\Gamma_{k}^{\wedge}$ to be the submodule of $\Gamma_{k}=D_{k}\left[Q_{k, 0}^{-1}\right]$ spanned by all monomials $\gamma=Q_{k, k-1}^{i_{k-1}} \cdots Q_{k, 0}^{i_{0}}$ with $i_{k-1}, \ldots, i_{1} \geq 0, i_{0} \in \mathbb{Z}$, and $i_{0}+\operatorname{deg} \gamma \geq 0$. He also shows in 20] that the homomorphism

$$
\begin{gathered}
\partial_{k}: \mathbb{F}_{2}\left[v_{1}^{ \pm 1}, \ldots, v_{k}^{ \pm 1}\right] \rightarrow \mathbb{F}_{2}\left[v_{1}^{ \pm 1}, \ldots, v_{k-1}^{ \pm 1}\right], \\
\partial_{k}\left(v_{1}^{j_{1}} \cdots v_{k}^{j_{k}}\right):= \begin{cases}v_{1}^{j_{1}} \cdots v_{k-1}^{j_{k-1}}, & \text { if } j_{k}=-1, \\
0, & \text { otherwise, }\end{cases}
\end{gathered}
$$

maps $\Gamma_{k}^{\wedge}$ to $\Gamma_{k-1}^{\wedge}$. Moreover, it is a differential on $\Gamma^{\wedge}=\bigoplus_{k} \Gamma_{k}^{\wedge}$. This module is bigraded by putting $\operatorname{bideg}\left(v_{1}^{j_{1}} \cdots v_{k}^{j_{k}}\right)=\left(k, k+\sum j_{i}\right)$.

Let $\Lambda$ be the (opposite) lambda algebra, in which the product in lambda symbols is written in the order opposite to that used in [3]. It is also bigraded by putting (as in [19, p. 90]) bideg $\left(\lambda_{i}\right)=(1,1+i)$. Singer proves in [20] that $\Gamma^{\wedge}$ is a differential bigraded coalgebra, which is dual to the differential bigraded lambda algebra $\Lambda$ via the isomorphisms

$$
\begin{array}{ccc}
\Gamma_{k}^{\wedge} & \rightarrow & \Lambda_{k}^{*} \\
v_{1}^{j_{1}} \cdots v_{k}^{j_{k}} & \mapsto & \left(\lambda_{j_{1}} \cdots \lambda_{j_{k}}\right)^{*} .
\end{array}
$$

Here the duality $*$ is taken with respect to the basis of admissible monomials of $\Lambda$. As a consequence, one gets an isomorphism of bigraded coalgebras

$$
H_{*}\left(\Gamma^{\wedge}\right) \cong \operatorname{Tor}_{*}^{\mathcal{A}}\left(\mathbb{F}_{2}, \mathbb{F}_{2}\right) \text {. }
$$

In the remaining part of this section, we recall the definition of the Lannes-Zarati homomorphism.

Let $P_{1}=\mathbb{F}_{2}[x]$ with $|x|=1$. Let $\hat{P} \subset \mathbb{F}_{2}\left[x, x^{-1}\right]$ be the submodule spanned by all powers $x^{i}$ with $i \geq-1$. The canonical $\mathcal{A}$-action on $P_{1}$ is extended to an $\mathcal{A}$-action on $\mathbb{F}_{2}\left[x, x^{-1}\right]$ (see Adams [2], Wilkerson [24]). Then $\hat{P}$ is an $\mathcal{A}$-submodule of $\mathbb{F}_{2}\left[x, x^{-1}\right]$. One has a short-exact sequence of $\mathcal{A}$-modules

\section{1.}

$$
0 \rightarrow P_{1} \stackrel{\iota}{\rightarrow} \hat{P} \stackrel{\pi}{\rightarrow} \Sigma^{-1} \mathbb{F}_{2} \rightarrow 0,
$$

where $\iota$ is the inclusion and $\pi$ is given by $\pi\left(x^{i}\right)=0$ if $i \neq-1$ and $\pi\left(x^{-1}\right)=1$. Let $e_{1}$ be the corresponding element in $\operatorname{Ext}_{\mathcal{A}}^{1}\left(\Sigma^{-1} \mathbb{F}_{2}, P_{1}\right)$.

Definition 2.2 (Singer [21]). (i) $e_{k}=\underbrace{e_{1} \otimes \cdots \otimes e_{1}}_{k \text { times }} \in \operatorname{Ext}_{\mathcal{A}}^{k}\left(\Sigma^{-k} \mathbb{F}_{2}, P_{k}\right)$.

(ii) $e_{k}(M)=e_{k} \otimes M \in \operatorname{Ext}_{\mathcal{A}}^{k}\left(\Sigma^{-k} M, P_{k} \otimes M\right)$, for $M$ a left $\mathcal{A}$-module.

Here $M$ also means the identity map of $M$.

Following Lannes-Zarati [14], the destabilization of $M$ is defined by

$$
\mathcal{D} M=M / E M,
$$

where $E M:=\operatorname{Span}\left\{S q^{i} x \mid i>\operatorname{deg} x, x \in M\right\}$. They show that the functor associating $M$ to $\mathcal{D} M$ is a right exact functor. Then they define $\mathcal{D}_{k}$ to be the $k$ th left derived functor of $\mathcal{D}$. So one gets

$$
\mathcal{D}_{k}(M)=H_{k}\left(\mathcal{D} F_{*}(M)\right)
$$


where $F_{*}(M)$ is an $\mathcal{A}$-free (or $\mathcal{A}$-projective) resolution of $M$.

The cap-product with $e_{k}(M)$ gives rise to the homomorphism

$$
\begin{aligned}
e_{k}(M): \mathcal{D}_{k}\left(\Sigma^{-k} M\right) & \rightarrow \mathcal{D}_{0}\left(P_{k} \otimes M\right) \equiv P_{k} \otimes M \\
e_{k}(M)(z) & =e_{k}(M) \cap z .
\end{aligned}
$$

Since $\mathbb{F}_{2}$ is an unstable $\mathcal{A}$-module, one gets

Theorem 2.3 (Lannes-Zarati [14). Let $D_{k} \subset P_{k}$ be the Dickson algebra of $k$ variables. Then $\alpha_{k}:=e_{k}\left(\Sigma \mathbb{F}_{2}\right): \mathcal{D}_{k}\left(\Sigma^{1-k} \mathbb{F}_{2}\right) \rightarrow \Sigma D_{k}$ is an isomorphism of internal degree 0.

By definition of the functor $\mathcal{D}$, one has a natural homomorphism, $\mathcal{D}(M) \rightarrow$ $\mathbb{F}_{2} \otimes M$. Then it induces a commutative diagram

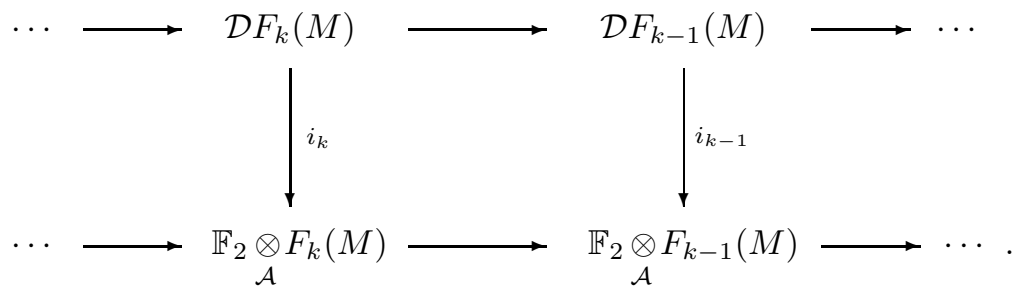

Here the horizontal arrows are induced from the differential in $F_{*}(M)$, and

$$
\left.i_{k}[Z]=\underset{\mathcal{A}}{1 \otimes Z}\right]
$$

for $Z \in F_{k}(M)$. Passing to homology, one gets a homomorphism

2.4 .

$$
\begin{array}{cccc}
i_{k}: & \mathbb{F}_{2} \otimes \mathcal{D}_{k}(M) & \rightarrow & \operatorname{Tor}_{k}^{\mathcal{A}}\left(\mathbb{F}_{2}, M\right) \\
& \mathcal{A}_{\mathcal{A}} \otimes[Z] & \mapsto & {[1 \underset{\mathcal{A}}{\otimes} Z] .} \\
& \mathcal{A}^{\prime} & &
\end{array}
$$

Taking $M=\Sigma^{1-k} \mathbb{F}_{2}$, one obtains a homomorphism

$$
i_{k}: \mathbb{F}_{2} \otimes \mathcal{D}_{k}\left(\Sigma^{1-k} \mathbb{F}_{2}\right) \rightarrow \operatorname{Tor}_{k}^{\mathcal{A}}\left(\mathbb{F}_{2}, \Sigma^{1-k} \mathbb{F}_{2}\right)
$$

Note that the suspension $\Sigma: \mathbb{F}_{2} \otimes D_{k} \rightarrow \mathbb{F}_{2} \otimes \Sigma D_{k}$ and the desuspension

$$
\Sigma^{-1}: \operatorname{Tor}_{k}^{\mathcal{A}}\left(\mathbb{F}_{2}, \Sigma^{1-k} \mathbb{F}_{2}\right) \stackrel{\cong}{\longrightarrow} \operatorname{Tor}_{k}^{\mathcal{A}}\left(\mathbb{F}_{2}, \Sigma^{-k} \mathbb{F}_{2}\right)
$$

are isomorphisms of internal degree 1 and $(-1)$, respectively. This leads one to

Definition 2.5 (Lannes-Zarati [14]). The homomorphism $\varphi_{k}$ of internal degree 0 is the dual of

$$
\varphi_{k}^{*}=\Sigma^{-1} i_{k}\left(1 \underset{\mathcal{A}}{\otimes} \alpha_{k}^{-1}\right) \Sigma: \mathbb{F}_{2} \otimes D_{\mathcal{A}} \rightarrow \operatorname{Tor}_{k}^{\mathcal{A}}\left(\mathbb{F}_{2}, \Sigma^{-k} \mathbb{F}_{2}\right) .
$$

Remark 2.6. In Theorem 3.9 we also denote by $\varphi_{k}^{*}$ the composite of the above $\varphi_{k}^{*}$ with the suspension isomorphism $\Sigma^{k}: \operatorname{Tor}_{k, i}^{\mathcal{A}}\left(\mathbb{F}_{2}, \Sigma^{-k} \mathbb{F}_{2}\right) \stackrel{\cong}{\longrightarrow} \operatorname{Tor}_{k, k+i}^{\mathcal{A}}\left(\mathbb{F}_{2}, \mathbb{F}_{2}\right)$.

We need to relate $\alpha_{k}=e_{k}\left(\Sigma \mathbb{F}_{2}\right)$ with connecting homomorphisms.

Suppose $f \in \operatorname{Ext}_{\mathcal{A}}^{1}\left(M_{3}, M_{1}\right)$ is represented by the short-exact sequence of left $\mathcal{A}$-modules $0 \rightarrow M_{1} \rightarrow M_{2} \rightarrow M_{3} \rightarrow 0$. Let $\Delta(f): \mathcal{D}_{s}\left(M_{3}\right) \rightarrow \mathcal{D}_{s-1}\left(M_{1}\right)$ be the connecting homomorphism associated with this short-exact sequence. Then one easily verifies

for any $z \in \mathcal{D}_{s}\left(M_{3}\right)$.

$$
\Delta(f)(z)=f \cap z
$$


One has

2.7. $e_{k}\left(\Sigma \mathbb{F}_{2}\right)=\left(e_{1}\left(\Sigma \mathbb{F}_{2}\right) \otimes P_{k-1}\right) \circ \cdots \circ\left(e_{1}\left(\Sigma^{3-k} \mathbb{F}_{2}\right) \otimes P_{1}\right) \circ e_{1}\left(\Sigma^{2-k} \mathbb{F}_{2}\right)$.

Therefore, one gets

2.8. $\alpha_{k}=\Delta\left(e_{1}\left(\Sigma \mathbb{F}_{2}\right) \otimes P_{k-1}\right) \circ \cdots \circ \Delta\left(e_{1}\left(\Sigma^{3-k} \mathbb{F}_{2}\right) \otimes P_{1}\right) \circ \Delta e_{1}\left(\Sigma^{2-k} \mathbb{F}_{2}\right)$.

(See Singer [21, p. 498].)

This formula will be useful to construct a chain-level representation of $\alpha_{k}$.

\section{A ChAin-LEVEl Representation OF THE LANNES-ZARATi HOMOMORPhism}

Suppose again $M$ is a left graded $\mathcal{A}$-module. Let $B_{*}(M)$ be the bar resolution of $M$ over $\mathcal{A}$. Recall that

$$
B_{k}(M)=\mathcal{A} \otimes \underbrace{I \otimes \cdots \otimes I}_{k \text { times }} \otimes M \quad(k \geq 0),
$$

where $I$ denotes the augmentation ideal of $\mathcal{A}$ and the tensor products are taken over $\mathbb{F}_{2}$. The module $B_{*}(M)=\bigoplus_{k} B_{k}(M)$ is bigraded by assigning an element $a_{0} \otimes a_{1} \otimes \cdots \otimes a_{k} \otimes x$ with homological degree $k$ and internal degree $\sum_{i=0}^{k}\left(\operatorname{deg} a_{i}\right)+$ $\operatorname{deg} x$.

The differential $d_{k}: B_{k}(M) \rightarrow B_{k-1}(M)$ is defined by

$$
\begin{aligned}
d_{k}\left(a_{0} \otimes a_{1} \otimes \cdots \otimes a_{k} \otimes x\right)= & a_{0} a_{1} \otimes \cdots \otimes a_{k} \otimes x+a_{0} \otimes a_{1} a_{2} \otimes \cdots \otimes a_{k} \otimes x \\
& +\cdots+a_{0} \otimes a_{1} \otimes \cdots \otimes a_{k} x .
\end{aligned}
$$

So $d_{k}$ preserves internal degree and lowers homological degree by 1 .

The action of $\mathcal{A}$ on $B_{k}(M)$ is given by

$$
a\left(a_{0} \otimes a_{1} \otimes \cdots \otimes a_{k} \otimes x\right)=a a_{0} \otimes a_{1} \otimes \cdots \otimes a_{k} \otimes x,
$$

for $a \in \mathcal{A}$.

Suppose additionally that $N$ is a right graded $\mathcal{A}$-module. As the bar resolution is an $\mathcal{A}$-free resolution, by definition one has

$$
\operatorname{Tor}_{k}^{\mathcal{A}}(N, M):=H_{k}\left(N \underset{\mathcal{A}}{\otimes} B_{*}(M)\right)
$$

Since $D_{k} \subset \mathbb{F}_{2}\left[v_{1}, \ldots, v_{k}\right]$, every element $q \in D_{k}$ has an unique expansion

$$
q=\sum_{\left(j_{1}, \ldots, j_{k}\right)} v_{1}^{j_{1}} \cdots v_{k}^{j_{k}}
$$

where $j_{1}, \ldots, j_{k}$ are non-negative. We associate with $q \in D_{k}$ the following element of internal degree $\sum_{i=1}^{k} j_{i}+1$ :

\section{Definition 3.1.}

$$
\tilde{q}=\sum_{\left(j_{1}, \ldots, j_{k}\right)} S q^{j_{1}+1} \otimes \cdots \otimes S q^{j_{k}+1} \otimes \Sigma^{1-k} 1 \in B_{k-1}\left(\Sigma^{1-k} \mathbb{F}_{2}\right)
$$

Lemma 3.2. If $q \in D_{k}$, then

$$
\tilde{q} \in E B_{k-1}\left(\Sigma^{1-k} \mathbb{F}_{2}\right):=\operatorname{Span}\left\{S q^{i} x \mid i>\operatorname{deg} x, x \in B_{k-1}\left(\Sigma^{1-k} \mathbb{F}_{2}\right)\right\} .
$$


Proof. From the definition of the $\mathcal{A}$-action on the bar resolution, one has

$$
S q^{j_{1}+1} \otimes \cdots \otimes S q^{j_{k}+1} \otimes \Sigma^{1-k} 1=S q^{j_{1}+1}\left(1 \otimes S q^{j_{2}+1} \otimes \cdots \otimes S q^{j_{k}+1} \otimes \Sigma^{1-k} 1\right) .
$$

Hence, it suffices to show that

$$
j_{1}+1>\left(j_{2}+1\right)+\cdots+\left(j_{k}+1\right)+(1-k)=j_{2}+\cdots+j_{k},
$$

for every term in the expansion of $\tilde{q}$.

Recall that $V_{i}=v_{1}^{2^{i-2}} v_{2}^{2^{i-3}} \cdots v_{i-1} v_{i}$. So, one easily verifies that every element $v \in M_{k}=\mathbb{F}_{2}\left[V_{1}, \ldots, V_{k}\right]$ is a sum of monomials $v_{1}^{j_{1}} \cdots v_{k}^{j_{k}}$, which satisfy the condition

$$
j_{1} \geq j_{2}+\cdots+j_{k} .
$$

The lemma follows from the fact that the Dickson algebra $D_{k}$ is a subalgebra of the Mùi algebra $M_{k}$.

Lemma 3.3. $\tilde{q}$ is a cycle in the chain complex $E B_{*}\left(\Sigma^{1-k} \mathbb{F}_{2}\right)$, for every $q \in D_{k}$.

This is a consequence of the following lemma, which is actually an exposition of the Adem relations.

Lemma 3.4. The homomorphism

$$
\begin{aligned}
\pi_{k, p}: \Delta_{k} & \rightarrow \mathcal{A}^{k-1}=\mathcal{A} \otimes \cdots \otimes \mathcal{A} \quad(k-1 \text { times }) \\
v_{1}^{j_{1}} \cdots v_{p}^{j_{p}} v_{p+1}^{j_{p+1}} \cdots v_{k}^{j_{k}} & \mapsto S q^{j_{1}+1} \otimes \cdots \otimes S q^{j_{p}+1} S q^{j_{p+1}+1} \otimes \cdots \otimes S q^{j_{k}+1}
\end{aligned}
$$

vanishes on $\Gamma_{k} \subset \Delta_{k}$, for $1 \leq p<k$.

Proof. Consider the diagonal $\psi: \Delta_{k} \rightarrow \Delta_{p-1} \otimes \Delta_{2} \otimes \Delta_{k-p-1}$ defined by

$$
\psi\left(v_{i}\right)= \begin{cases}v_{i} \otimes 1 \otimes 1, & i<p, \\ 1 \otimes v_{i-p+1} \otimes 1, & p \leq i \leq p+1, \\ 1 \otimes 1 \otimes v_{i-p-1}, & p+1<i\end{cases}
$$

From Proposition 2.1 of Singer 20, one gets

$$
\psi\left(\Gamma_{k}\right) \subset \Gamma_{p-1} \otimes \Gamma_{2} \otimes \Gamma_{k-p-1} .
$$

Define the homomorphism $\omega_{t}: \Gamma_{t} \rightarrow \mathcal{A}^{t}$ by

$$
\omega_{t}\left(v_{1}^{j_{1}} \cdots v_{t}^{j_{t}}\right)=S q^{j_{1}+1} \otimes \cdots \otimes S q^{j_{t}+1} .
$$

Then one has

$$
\pi_{k, p}=\left(\omega_{p-1} \otimes \pi_{2,1} \otimes \omega_{k-p-1}\right) \psi .
$$

By Proposition 3.1 of Singer [20], the Adem relations yield

$$
\pi_{2,1}\left(\Gamma_{2}\right)=0 \text {. }
$$

Hence, $\pi_{k, p}\left(\Gamma_{k}\right)=0$ for $1 \leq p<k$. The lemma is proved.

Proof of Lemma 3.3. First, we note that $S q^{j_{k}+1}\left(\Sigma^{1-k} 1\right)=0$ for any $j_{k} \geq 0$. Then, by definition of the differential in the bar resolution, we get

$$
d_{k-1}(\tilde{q})=\sum_{p=1}^{k-1}\left(\pi_{k, p} \otimes i d_{\Sigma^{1-k} \mathbb{F}_{2}}\right)\left(q \otimes \Sigma^{1-k} 1\right) .
$$

Since $q \in D_{k} \subset \Gamma_{k}$, Lemma 3.4 yields $\pi_{k, p}(q)=0$. Thus $d_{k-1}(\tilde{q})=0$. The lemma is proved. 
For the convenience of the latter use, we define $\tilde{\pi}_{k, p}$ as follows:

$$
\tilde{\pi}_{k, p}\left(S q^{j_{1}+1} \otimes \cdots \otimes S q^{j_{k}+1}\right)=S q^{j_{1}+1} \otimes \cdots \otimes S q^{j_{p}+1} S q^{j_{p+1}+1} \otimes \cdots \otimes S q^{j_{k}+1}
$$

for $1 \leq p<k$.

Suppose as before that

$$
q=\sum_{J=\left(j_{1}, \ldots, j_{k}\right)} v_{1}^{j_{1}} \cdots v_{k}^{j_{k}} \in D_{k}
$$

For a fixed $(k-s)$-index $\left(j_{s+1}, \ldots, j_{k}\right)$, we define $J\left(j_{s+1}, \ldots, j_{k}\right)$ to be the set of all $s$ indices $\left(j_{1}, \ldots, j_{s}\right)$ 's such that $\left(j_{1}, \ldots, j_{s}, j_{s+1}, \ldots, j_{k}\right)$ occurs as a $k$-index in the above sum.

The following lemma is a slight generalization of Lemma 3.4

Lemma 3.5. If $q=\sum_{J} v_{1}^{j_{1}} \cdots v_{k}^{j_{k}} \in D_{k}$, then

$$
\tilde{\pi}_{s, p}\left(\sum_{J\left(j_{s+1}, \ldots, j_{k}\right)} S q^{j_{1}+1} \otimes \cdots \otimes S q^{j_{s}+1}\right)=0
$$

for $1 \leq p<s \leq k$.

Proof. Let us consider the diagonal $\psi_{2}: \Delta_{k} \rightarrow \Delta_{s} \otimes \Delta_{k-s}$ given by

$$
\psi_{2}\left(v_{i}\right)= \begin{cases}v_{i} \otimes 1, & 1 \leq i \leq s, \\ 1 \otimes v_{i-s}, & s<i \leq k .\end{cases}
$$

According to Proposition 2.1 of Singer [20, $\psi\left(\Gamma_{k}\right) \subset \Gamma_{s} \otimes \Gamma_{k-s}$. Since $q \in D_{k} \subset \Gamma_{k}$, it implies $\sum_{J\left(j_{s+1}, \ldots, j_{k}\right)} v_{1}^{j_{1}} \cdots v_{s}^{j_{s}} \in \Gamma_{s}$. Then, by Lemma 3.4, we have

$$
\tilde{\pi}_{s, p}\left(\sum_{J\left(j_{s+1}, \ldots, j_{k}\right)} S q^{j_{1}+1} \otimes \cdots \otimes S q^{j_{s}+1}\right)=\pi_{s, p}\left(\sum_{J\left(j_{s+1}, \ldots, j_{k}\right)} v_{1}^{j_{1}} \cdots v_{s}^{j_{s}}\right)=0 .
$$

The lemma is proved.

By definition of the destabilization functor $\mathcal{D}$, for any left $\mathcal{A}$-module $M$, one has an exact sequence of chain complexes

$$
0 \rightarrow E B_{*}(M) \stackrel{i_{E}}{\longrightarrow} B_{*}(M) \stackrel{j_{\mathcal{D}}}{\longrightarrow} \mathcal{D} B_{*}(M) \rightarrow 0,
$$

in which the bar resolution $B_{*}(M)$ is exact. Hence, by use of the induced long exact sequence, the connecting homomorphism is an isomorphism

$$
\partial_{*}: \mathcal{D}_{k}(M):=H_{k}\left(\mathcal{D} B_{*}(M)\right) \stackrel{\cong}{\longrightarrow} H_{k-1}\left(E B_{*}(M)\right) .
$$

Take $M=\Sigma^{1-k} \mathbb{F}_{2}$. The following lemma deals with the connecting isomorphism

$$
\partial_{*}: \mathcal{D}_{k}\left(\Sigma^{1-k} \mathbb{F}_{2}\right):=H_{k}\left(\mathcal{D} B_{*}\left(\Sigma^{1-k} \mathbb{F}_{2}\right)\right) \stackrel{\cong}{\longrightarrow} H_{k-1}\left(E B_{*}\left(\Sigma^{1-k} \mathbb{F}_{2}\right)\right) .
$$

Let $[\tilde{q}]$ be the homology class of the cycle $\tilde{q}$ in

$$
\mathcal{D}_{k}\left(\Sigma^{1-k} \mathbb{F}_{2}\right) \cong H_{k-1}\left(E B_{*}\left(\Sigma^{1-k} \mathbb{F}_{2}\right)\right) \text {. }
$$

Lemma 3.6. If $q \in D_{k}$, then

$$
\partial_{*}[1 \otimes \tilde{q}]=[\tilde{q}] .
$$

Proof. Suppose $q=\sum_{J} v_{1}^{j_{1}} \cdots v_{k}^{j_{k}}$. The element $\sum_{J} 1 \otimes S q^{j_{1}+1} \otimes \cdots \otimes S q^{j_{k}+1} \otimes$ $\Sigma^{1-k} 1 \in B_{k}\left(\Sigma^{1-k} \mathbb{F}_{2}\right)$ is a lifting over $j_{\mathcal{D}}$ of its class modulo $E B_{k}\left(\Sigma^{1-k} \mathbb{F}_{2}\right)$ in $\mathcal{D} B_{k}\left(\Sigma^{1-k} \mathbb{F}_{2}\right)$. Let $d$ denote the differential in $B_{*}\left(\Sigma^{1-k} \mathbb{F}_{2}\right)$, we get 
SPHERICAL CLASSES AND THE LAMBDA ALGEBRA

$$
\begin{aligned}
& d\left(\sum_{J} 1 \otimes S q^{j_{1}+1} \otimes \cdots \otimes S q^{j_{k}+1} \otimes \Sigma^{1-k} 1\right) \\
&= \sum_{J} 1 \cdot S q^{j_{1}+1} \otimes \cdots \otimes S q^{j_{k}+1} \otimes \Sigma^{1-k} 1 \\
&+\sum_{p=1}^{k-1} 1 \otimes \tilde{\pi}_{k, p}\left(\sum_{J} S q^{j_{1}+1} \otimes \cdots \otimes S q^{j_{k}+1}\right) \otimes \Sigma^{1-k} 1 \\
&+\sum_{J} 1 \otimes S q^{j_{1}+1} \otimes \cdots \otimes S q^{j_{k}+1} \Sigma^{1-k} 1 .
\end{aligned}
$$

By Lemma 3.4

$$
\tilde{\pi}_{k, p}\left(\sum_{J} S q^{j_{1}+1} \otimes \cdots \otimes S q^{j_{k}+1}\right)=\pi_{k, p}(q)=0 .
$$

On the other hand, $S q^{j_{k}+1}\left(\Sigma^{1-k} 1\right)=0$ for any $j_{k} \geq 0$. Therefore, we obtain

$$
\begin{aligned}
d\left(\sum_{J} 1 \otimes S q^{j_{1}+1} \otimes \cdots \otimes S q^{j_{k}+1} \otimes \Sigma^{1-k} 1\right) & =\sum_{J} S q^{j_{1}+1} \otimes \cdots \otimes S q^{j_{k}+1} \otimes \Sigma^{1-k} 1 \\
& =i_{E}(\tilde{q}) .
\end{aligned}
$$

By definition of the connecting homomorphism, we have

$$
\partial_{*}[1 \otimes \tilde{q}]=[\tilde{q}] .
$$

The lemma is proved.

The following theorem deals with the isomorphism $\alpha_{k}: \mathcal{D}_{k}\left(\Sigma^{1-k} \mathbb{F}_{2}\right) \rightarrow \Sigma D_{k}$ treated in Theorem 2.3

Theorem 3.7. If $q \in D_{k}$, then

$$
\alpha_{k}[\tilde{q}]=\Sigma q .
$$

Proof. We compute $\alpha_{k}$ by means of the following formula

$$
\begin{aligned}
\alpha_{k} & =\Delta\left(e_{1}\left(\Sigma \mathbb{F}_{2}\right) \otimes P_{k-1}\right) \circ \cdots \circ \Delta\left(e_{1}\left(\Sigma^{3-k} \mathbb{F}_{2}\right) \otimes P_{1}\right) \circ \Delta e_{1}\left(\Sigma^{2-k} \mathbb{F}_{2}\right) \\
& =\delta_{k} \cdots \delta_{2} \delta_{1} .
\end{aligned}
$$

Here $\delta_{s}$ stands for $\Delta\left(e_{1}\left(\Sigma^{1-k+s} \mathbb{F}_{2}\right) \otimes P_{s-1}\right)$, for brevity.

Consider the short exact sequence representing $e_{1}\left(\Sigma^{2-k} \mathbb{F}_{2}\right)$ :

$$
0 \rightarrow \Sigma^{2-k} P_{1} \stackrel{\iota}{\rightarrow} \Sigma^{2-k} \hat{P} \stackrel{\pi}{\rightarrow} \Sigma^{1-k} \mathbb{F}_{2} \rightarrow 0 .
$$

Then the connecting homomorphism induced by this exact sequence is nothing but

$$
\delta_{1}: H_{k-1}\left(E B_{*}\left(\Sigma^{1-k} \mathbb{F}_{2}\right)\right) \rightarrow H_{k-2}\left(E B_{*}\left(\Sigma^{2-k} P_{1}\right)\right) .
$$

A lifting of $\tilde{q}=\sum_{J} S q^{j_{1}+1} \otimes \cdots \otimes S q^{j_{k}+1} \otimes \Sigma^{1-k} 1$ over $\pi$ is

$$
\sum_{J} S q^{j_{1}+1} \otimes \cdots \otimes S q^{j_{k}+1} \otimes \Sigma^{2-k} x_{k}^{-1} \in E B_{*}\left(\Sigma^{2-k} \hat{P}\right),
$$

where we are writing $P_{1}=\mathbb{F}_{2}\left[x_{k}\right], \hat{P}=\operatorname{Span}\left\{x_{k}^{i} \mid i \geq-1\right\}$. The boundary of this element in $E B_{*}\left(\Sigma^{2-k} \hat{P}\right)$ is pulled back under $\iota$ to a cycle in $E B_{*}\left(\Sigma^{2-k} P_{1}\right)$, which 
represents $\delta_{1}[\tilde{q}]$. That means

$$
\begin{aligned}
\delta_{1}[\tilde{q}]= & {\left[d\left(\sum_{J} S q^{j_{1}+1} \otimes \cdots \otimes S q^{j_{k}+1} \otimes \Sigma^{2-k} x_{k}^{-1}\right)\right] } \\
= & {\left[\sum_{p=1}^{k-1} \tilde{\pi}_{k, p}\left(\sum_{J} S q^{j_{1}+1} \otimes \cdots \otimes S q^{j_{k}+1}\right) \otimes \Sigma^{2-k} x_{k}^{-1}\right.} \\
& \left.+\sum_{J} S q^{j_{1}+1} \otimes \cdots \otimes S q^{j_{k-1}+1} \otimes S q^{j_{k}+1}\left(\Sigma^{2-k} x_{k}^{-1}\right)\right] \\
= & {\left[\sum_{J} S q^{j_{1}+1} \otimes \cdots \otimes S q^{j_{k-1}+1} \otimes \Sigma^{2-k} S q^{j_{k}+1}\left(x_{k}^{-1}\right)\right], }
\end{aligned}
$$

where the last equality follows from Lemma 3.4. Indeed,

$$
\tilde{\pi}_{k, p}\left(\sum_{J} S q^{j_{1}+1} \otimes \cdots \otimes S q^{j_{k}+1}\right)=\pi_{k, p}(q)=0 .
$$

Similarly, $\delta_{2}: H_{k-2}\left(E B_{*}\left(\Sigma^{2-k} P_{1}\right)\right) \rightarrow H_{k-3}\left(E B_{*}\left(\Sigma^{3-k} P_{2}\right)\right)$ is the connecting homomorphism induced by the short exact sequence representing $e_{1}\left(\Sigma^{3-k} \mathbb{F}_{2}\right) \otimes P_{1}$ :

$$
0 \rightarrow \Sigma^{3-k} P_{2} \stackrel{\iota \otimes P_{1}}{\longrightarrow} \Sigma^{3-k}\left(\hat{P} \otimes P_{1}\right) \stackrel{\pi \otimes P_{1}}{\longrightarrow} \Sigma^{2-k} P_{1} \rightarrow 0 .
$$

Here we are writing $P_{1}=\mathbb{F}_{2}\left[x_{k}\right], P_{2}=\mathbb{F}_{2}\left[x_{k-1}, x_{k}\right], \hat{P}=\operatorname{Span}\left\{x_{k-1}^{i} \mid i \geq-1\right\}$. A lifting of $S q^{j_{1}+1} \otimes \cdots \otimes S q^{j_{k-1}+1} \otimes \Sigma^{2-k} S q^{j_{k}+1}\left(x_{k}^{-1}\right)$ over $\pi \otimes P_{1}$ is

$$
S q^{j_{1}+1} \otimes \cdots \otimes S q^{j_{k-1}+1} \otimes \Sigma^{3-k} x_{k-1}^{-1} S q^{j_{k}+1}\left(x_{k}^{-1}\right) .
$$

Therefore, by an argument similar to the one given above, we get

$$
\begin{aligned}
\delta_{2} \delta_{1}[\tilde{q}]= & {\left[d\left(\sum_{J} S q^{j_{1}+1} \otimes \cdots \otimes S q^{j_{k-1}+1} \otimes \Sigma^{3-k} x_{k-1}^{-1} S q^{j_{k}+1}\left(x_{k}^{-1}\right)\right)\right] } \\
= & {\left[\sum_{p=1}^{k-2} \sum_{J} \tilde{\pi}_{k-1, p}\left(S q^{j_{1}+1} \otimes \cdots \otimes S q^{j_{k-1}+1}\right) \otimes \Sigma^{3-k} x_{k-1}^{-1} S q^{j_{k}+1}\left(x_{k}^{-1}\right)\right.} \\
& \left.+\sum_{J} S q^{j_{1}+1} \otimes \cdots \otimes S q^{j_{k-2}+1} \otimes S q^{j_{k-1}+1}\left(\Sigma^{3-k} x_{k-1}^{-1} S q^{j_{k}+1}\left(x_{k}^{-1}\right)\right)\right] \\
= & {\left[\sum_{J} S q^{j_{1}+1} \otimes \cdots \otimes S q^{j_{k-2}+1} \otimes \Sigma^{3-k} S q^{j_{k-1}+1}\left(x_{k-1}^{-1} S q^{j_{k}+1}\left(x_{k}^{-1}\right)\right)\right] }
\end{aligned}
$$

(by Lemma 3.5).

Repeating the above argument, we then have

$$
\begin{aligned}
\alpha_{k}[\tilde{q}] & =\delta_{k} \cdots \delta_{1}[\tilde{q}] \\
& =\left[\sum_{J}\left(\Sigma S q^{j_{1}+1}\left(x_{1}^{-1} S q^{j_{2}+1}\left(x_{2}^{-1} \cdots S q^{j_{k}+1}\left(x_{k}^{-1}\right) \cdots\right)\right)\right)\right] .
\end{aligned}
$$

By Theorem 3.2 of our paper [10], we get

$$
\left[\sum_{J}\left(\Sigma S q^{j_{1}+1}\left(x_{1}^{-1} S q^{j_{2}+1}\left(x_{2}^{-1} \cdots S q^{j_{k}+1}\left(x_{k}^{-1}\right) \cdots\right)\right)\right)\right]=[\Sigma q]=\Sigma q .
$$

The theorem is proved.

This theorem has an immediate consequence as follows. 
Corollary 3.8. The homomorphism $D_{k} \rightarrow E B_{k-1}\left(\Sigma^{1-k} \mathbb{F}_{2}\right), q \mapsto \tilde{q}$ is a chain-level representation of the homomorphism

$$
\left(1 \underset{\mathcal{A}}{\otimes} \alpha_{k}^{-1}\right) \Sigma: \mathbb{F}_{2} \otimes D_{\mathcal{A}} D_{k} \rightarrow \underset{\mathcal{A}}{\mathbb{F}_{2}} \otimes \mathcal{D}_{k}\left(\Sigma^{1-k} \mathbb{F}_{2}\right) .
$$

Theorem 3.9. The inclusion $D_{k} \subset \Gamma_{k}^{\wedge}$ is a chain-level representation of the LannesZarati dual homomorphism

$$
\varphi_{k}^{*}:\left(\mathbb{F}_{2} \underset{\mathcal{A}}{\otimes} D_{k}\right)_{i} \rightarrow \operatorname{Tor}_{k, k+i}^{\mathcal{A}}\left(\mathbb{F}_{2}, \mathbb{F}_{2}\right) .
$$

Proof. Suppose again that

$$
q=\sum_{J=\left(j_{1}, \ldots, j_{k}\right)} v_{1}^{j_{1}} \cdots v_{k}^{j_{k}} \in D_{k} .
$$

By Corollary 3.8 and Lemma 3.6, we have

$$
\begin{aligned}
& \left(1 \underset{\mathcal{A}}{\otimes} \alpha_{k}^{-1}\right) \Sigma: \quad \mathbb{F}_{2} \underset{\mathcal{A}}{\otimes} D_{k} \quad \rightarrow \quad \mathbb{F}_{2} \underset{\mathcal{A}}{\otimes \mathcal{D}_{k}}\left(\Sigma^{1-k} \mathbb{F}_{2}\right) \\
& {[q] \quad \mapsto[\tilde{q}] \stackrel{\partial_{*}}{=}[1 \otimes \tilde{q}] .}
\end{aligned}
$$

From the definition of $i_{k}$ (see 2.4), we get

$$
\begin{aligned}
i_{k}: \quad \mathbb{F}_{2} \otimes H_{k}\left(\mathcal{D} B_{*}\left(\Sigma^{1-k} \mathbb{F}_{2}\right)\right) & \rightarrow \operatorname{Tor}_{k}^{\mathcal{A}}\left(\mathbb{F}_{2}, \Sigma^{1-k} \mathbb{F}_{2}\right) \\
{[1 \otimes \tilde{q}] } & \mapsto[1 \otimes \tilde{q}] .
\end{aligned}
$$

Let us consider the desuspension

$$
\Sigma^{-1}: \operatorname{Tor}_{k}^{\mathcal{A}}\left(\mathbb{F}_{2}, \Sigma^{1-k} \mathbb{F}_{2}\right) \rightarrow \operatorname{Tor}_{k}^{\mathcal{A}}\left(\mathbb{F}_{2}, \Sigma^{-k} \mathbb{F}_{2}\right),
$$

which sends $\left[\sum_{J} 1 \otimes S q^{j_{1}+1} \otimes \cdots \otimes S q^{j_{k}+1} \otimes \Sigma^{1-k} 1\right]$ to $\left[\sum_{J} 1 \otimes S q^{j_{1}+1} \otimes \cdots \otimes\right.$ $\left.S q^{j_{k}+1} \otimes \Sigma^{-k} 1\right]$. Then the map

$$
\varphi_{k}^{*}=\Sigma^{-1} i_{k}\left(1 \underset{\mathcal{A}}{\otimes} \alpha_{k}^{-1}\right) \Sigma: \underset{\mathcal{A}}{\mathbb{F}_{2}} \underset{\mathcal{A}}{\otimes} D_{k} \rightarrow \operatorname{Tor}_{k}^{\mathcal{A}}\left(\mathbb{F}_{2}, \Sigma^{-k} \mathbb{F}_{2}\right)
$$

is given by

$$
\varphi_{k}^{*}[q]=\left[\sum_{J} 1 \otimes S q^{j_{1}+1} \otimes \cdots \otimes S q^{j_{k}+1} \otimes \Sigma^{-k} 1\right] .
$$

The canonical isomorphism

$$
\Sigma^{k}: \operatorname{Tor}_{k, i}^{\mathcal{A}}\left(\mathbb{F}_{2}, \Sigma^{-k} \mathbb{F}_{2}\right) \rightarrow \operatorname{Tor}_{k, k+i}^{\mathcal{A}}\left(\mathbb{F}_{2}, \mathbb{F}_{2}\right)
$$

is defined by the chain-level version

$$
\Sigma^{k}\left(a_{0} \otimes a_{1} \otimes \cdots \otimes a_{k} \otimes \Sigma^{-k} 1\right)=a_{0} \otimes a_{1} \otimes \cdots \otimes a_{k} \otimes 1 .
$$

By ambiguity of notation, the composite $\Sigma^{k} \varphi_{k}^{*}$ is also denoted by $\varphi_{k}^{*}$ (see Remark 2.6). Hence

$$
\begin{aligned}
\varphi_{k}^{*}:\left(\mathbb{F}_{2} \otimes D_{k}\right)_{i} & \rightarrow \operatorname{Tor}_{k, k+i}^{\mathcal{A}}\left(\mathbb{F}_{2}, \mathbb{F}_{2}\right) \\
{[q] } & \mapsto\left[\sum_{J} 1 \otimes S q^{j_{1}+1} \otimes \cdots \otimes S q^{j_{k}+1} \otimes 1\right] .
\end{aligned}
$$

In [18], Priddy constructs the Koszul complex $\bar{K}_{*}(\mathcal{A})$, a subcomplex of $B_{*}\left(\mathbb{F}_{2}\right)$, which is isomorphic to the dual of the lambda algebra. More precisely, it is defined as follows. Let $\Lambda$ be the (opposite) lambda algebra, in which the product in lambda symbols is written in the order opposite to that used in [3]. (See Singer [20, p. 687] 
for a precise definition of $\Lambda$.) Then, according to Priddy [18, $\S 7], \bar{K}_{*}(\mathcal{A})$ is the image of the monomorphism

$$
\begin{aligned}
\Lambda^{*} & \rightarrow B_{*}\left(\mathbb{F}_{2}\right) \\
\left(\lambda_{j_{1}} \cdots \lambda_{j_{k}}\right)^{*} & \mapsto 1 \otimes S q^{j_{1}+1} \otimes \cdots \otimes S q^{j_{k}+1} \otimes 1,
\end{aligned}
$$

which is a homotopy equivalence. Here $\Lambda^{*}$ denotes the dual of $\Lambda$ and the duality* is taken with respect to the basis of admissible monomials of $\Lambda$. Combining it with Singer's isomorphism

$$
\begin{aligned}
\Gamma^{\wedge} & \rightarrow \Lambda^{*} \\
v_{1}^{j_{1}} \cdots v_{k}^{j_{k}} & \mapsto\left(\lambda_{j_{1}} \cdots \lambda_{j_{k}}\right)^{*},
\end{aligned}
$$

we get the following homotopy equivalence

$$
\begin{aligned}
\Gamma^{\wedge} & \rightarrow B_{*}\left(\mathbb{F}_{2}\right) \\
v_{1}^{j_{1}} \cdots v_{k}^{j_{k}} & \mapsto 1 \otimes S q^{j_{1}+1} \otimes \cdots \otimes S q^{j_{k}+1} \otimes 1 .
\end{aligned}
$$

As a consequence, for any $q \in D_{k}$, we obtain

$$
\begin{aligned}
\varphi_{k}^{*}[q] & =\left[\sum_{J} 1 \otimes S q^{j_{1}+1} \otimes \cdots \otimes S q^{j_{k}+1} \otimes 1\right] \\
& =\left[\sum_{J} v_{1}^{j_{1}} \cdots v_{k}^{j_{k}}\right] \\
& =[q] .
\end{aligned}
$$

It means that the inclusion $D_{k} \subset \Gamma_{k}^{\wedge}$ is a chain-level representation of $\varphi_{k}^{*}$. The theorem is completely proved.

Corollary 3.10. Conjecture 1.2 is equivalent to Conjecture 1.3.

This follows immediately from Theorem 3.9 ,

We have proved Conjecture 1.2 for $k=3$ in 8 and Conjecture 1.3 for $k=3$ in [10].

\section{Factoring the Lannes-Zarati homomorphism}

The purpose of this section is to prove the following proposition.

Proposition 4.1. $\varphi_{k}^{*}$ factors through $\mathbb{F}_{2} \underset{\mathcal{A}}{\otimes}$ Ker $\partial_{k}$ as shown in the commutative diagram:

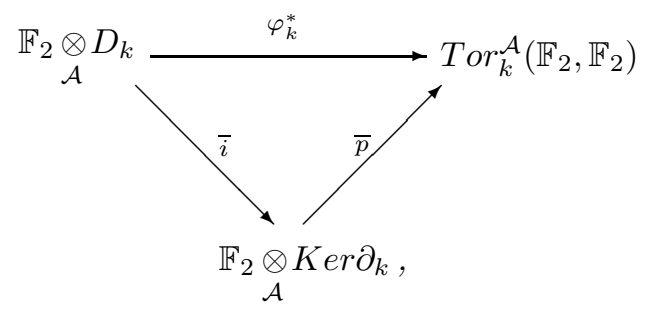

where $\bar{i}$ is induced by the inclusion $D_{k} \subset K e r \partial_{k}$, and $\bar{p}$ is an epimorphism induced by the canonical projection $p: \operatorname{Ker}_{k} \rightarrow H_{k}\left(\Gamma^{\wedge}\right) \cong \operatorname{Tor}_{k}^{\mathcal{A}}\left(\mathbb{F}_{2}, \mathbb{F}_{2}\right)$.

Proof. The canonical projection

$$
p: \operatorname{Ker}_{k} \rightarrow \operatorname{Tor}_{k}^{\mathcal{A}}\left(\mathbb{F}_{2}, \mathbb{F}_{2}\right)=\operatorname{Ker}_{k} / \operatorname{Im}_{k+1}
$$

sends $x$ to $[x]=x+\operatorname{Im} \partial_{k+1}$. 
By Theorem 5.15 of Singer [20], the action of $\mathcal{A}$ on $K e r \partial_{k}$ induces a trivial action of $\mathcal{A}$ upon $\operatorname{Tor}_{k}^{\mathcal{A}}\left(\mathbb{F}_{2}, \mathbb{F}_{2}\right)$. Therefore, $p$ induces the epimorphism

$$
\begin{aligned}
\bar{p}: \mathbb{F}_{2} \otimes \operatorname{Ker}_{k} & \rightarrow \operatorname{Tor}_{k}^{\mathcal{A}}\left(\mathbb{F}_{2}, \mathbb{F}_{2}\right) \\
{[x] } & \mapsto[x] .
\end{aligned}
$$

For any $q \in D_{k}$, we have

$$
\bar{p} \cdot \bar{i}[q]=\bar{p}[q]=[q]=\varphi_{k}^{*}[q] .
$$

So, we get $\varphi_{k}^{*}=\bar{p} \cdot \bar{i}$. The proposition is proved.

In [10, we have stated the following conjecture.

Conjecture 4.2. $D_{k}^{+} \subset \mathcal{A}^{+} \cdot K \operatorname{Ker} \partial_{k}$ for $k>2$.

Obviously, this is stronger than Conjectures 1.2 and 1.3 and equivalent to the following one.

Conjecture 4.3. The homomorphism $\bar{i}: \mathbb{F}_{2} \otimes D_{k} \rightarrow \mathbb{F}_{2} \otimes K e r \partial_{k}$, induced by the inclusion $i: D_{k} \rightarrow K e r \partial_{k}$, is trivial for $k>2$. $^{\mathcal{A}}$

Based on the above discussion, we believe the following problem is something of interest.

Problem 4.4. Determine $\mathbb{F}_{2} \otimes K e r \partial_{k}$.

$\mathcal{A}$

\section{REFERENCES}

[1] J. F. Adams, On the non-existence of elements of Hopf invariant one, Ann. Math. 72 (1960), 20-104. MR 25:4530

[2] J. F. Adams, Operations of the nth kind in K-theory and what we don't know about $R P^{\infty}$, New developments in topology, G. Segal (ed.), London Math. Soc. Lect. Note Series 11 (1974), 1-9. MR 49:3941

[3] A. K. Bousfield, E. B. Curtis, D. M. Kan, D. G. Quillen, D. L. Rector, J. W. Schlesinger, The mod $p$ lower central series and the Adams spectral sequence, Topology 5 (1966), 331-342. MR 33:8002

[4] W. Browder, The Kervaire invariant of a framed manifold and its generalization, Ann. Math. 90 (1969), 157-186. MR 40:4963

[5] E. B. Curtis, The Dyer-Lashof algebra and the $\Lambda$-algebra, Illinois Jour. Math. 19 (1975), 231-246. MR 51:14054

[6] L. E. Dickson, A fundamental system of invariants of the general modular linear group with a solution of the form problem, Trans. Amer. Math. Soc. 12 (1911), 75-98. CMP 95:18

[7] P. G. Goerss, Unstable projectives and stable Ext : with applications, Proc. London Math. Soc. 53 (1986), 539-561. MR 88d:55011

[8] N. H. V. Hu'ng, Spherical classes and the algebraic transfer, Trans. Amer. Math. Soc. 349 (1997), 3893-3910. MR 98e:55020

[9] N. H. V. Hu'ng, Spherical classes and the homology of the Steenrod algebra, Vietnam Jour. Math. 26 (1998), 373-377.

[10] N. H. V. Hu'ng, The weak conjecture on spherical classes, Math. Zeit. 231 (1999), 727-743. MR 2000g:55019

[11] N. H. V. Hu'ng and F. P. Peterson, Spherical classes and the Dickson algebra, Math. Proc. Camb. Phil. Soc. 124 (1998), 253-264. MR 99i:55021

[12] J. Lannes, Sur le n-dual du n-ème spectre de Brown-Gitler, Math. Zeit. 199 (1988), 29-42. MR 89h:55020

[13] J. Lannes and S. Zarati, Invariants de Hopf d'ordre supérieur et suite spectrale d'Adams, C. R. Acad. Sci. 296 (1983), 695-698. MR 85a:55009

[14] J. Lannes and S. Zarati, Sur les foncteurs dérivés de la déstabilisation, Math. Zeit. 194 (1987), 25-59. MR 88j:55014 
[15] S. Mac Lane, Homology, Die Grundlehren der Math. Wissenschaften, Band 114, Academic Press, Springer-Verlag, Berlin and New York, 1963. MR 28:122]

[16] I. Madsen, On the action of the Dyer-Lashof algebra in $H_{*}(G)$, Pacific Jour. Math. 60 (1975), 235-275. MR 52:9228

[17] H. Mùi, Modular invariant theory and cohomology algebras of symmetric groups, Jour. Fac. Sci. Univ. Tokyo, 22 (1975), 310-369. MR 54:10440

[18] S. B. Priddy, Koszul resolutions, Trans. Amer. Math. Soc. 152 (1970), 39-60. MR 42:346

[19] D. C. Ravenel, Complex cobordism and stable homotopy groups of spheres, Academic Press, 1986. MR 87j:55003

[20] W. M. Singer, Invariant theory and the lambda algebra, Trans. Amer. Math. Soc. 280 (1983), 673-693. MR 85e:55029

[21] W. M. Singer, The transfer in homological algebra, Math. Zeit. 202 (1989), 493-523. MR 90i:55035

[22] V. Snaith and J. Tornehave, On $\pi_{*}^{S}(B O)$ and the Arf invariant of framed manifolds, Amer. Math. Soc. Contemporary Math. 12 (1982), 299-313. MR 83k:55008

[23] R. J. Wellington, The unstable Adams spectral sequence of free iterated loop spaces, Memoirs Amer. Math. Soc. 258 (1982). MR 83c:55028

[24] C. Wilkerson, Classifying spaces, Steenrod operations and algebraic closure, Topology 16 (1977), 227-237. MR 56:1307

Department of Mathematics, Vietnam National University, Hanoi, 334 Nguyên Trã Street, Hanoi, Vietnam

E-mail address: nhvhung@hotmail.com 